\title{
Histochemical studies on various dehydrogenases related to glucose metabolism of the human skin
}

\author{
Takenori Hashimoto,
}

(Department of Dermatology, Osaka University School of Medicine)

In the present investigation, the distribution of various dehydrogenases related to glucose metabolism in the human skin was examined microscopically using Nitro-blue tetrazolium at the cellular level, and the relation of the glucose metabolism to the minute morphology of the human skin was discussed. The different dehydrogenases $(\mathrm{DH})$ tested are glyceraldehyde-3-phosphate $\mathrm{DH}$ (GA3PDH) and lactic $\mathrm{DH}(\mathrm{LDH})$ of the Embden-Meyerhof pathway; DPNlinked isocitric DH (DPN-ICDH), TPH-linked isocitric DH (TPN-ICDH), succinic $\mathrm{DH}(\mathrm{SDH})$ and malic $\mathrm{DH}(\mathrm{MDH})$ of the TCA cycle; glucose-6-phosphate $\mathrm{DH}(\mathrm{G} 6 \mathrm{PDH})$ and 6-phosphogluconic DH $(6 \mathrm{PGDH})$ of the hexose monophosphate shunt; Ochoa's malic enzyme (ME), glutamic $\mathrm{DH}(\mathrm{GDH})$ and $\beta$-hydroxybutyric $\mathrm{DH}(\boldsymbol{\beta}-\mathrm{HBDH})$ as enzymes close to the TCA cycle; DPN-linked L-gulonic DH (DPN-L-GuDH) and TPN-linked L-gulonic DH (TPN-L-GuDH) of the glucuronic acid cycle; DPNH-tetrazolium reductase (DPNH-TR) and TPNH-tetrazolium reductase (TPNH-TR).

\section{Materials and methods}

Pieces of skin were surgically excised from the scalp, trunk, buttock, axilla and sole of healthy human adults and were cut $20 \mu$ thick sections in a cryostat at $-20^{\circ} \mathrm{C}$, mounted on glass slides and finger-thawed. The sections were inserted into an incubation medium, incubated at $37^{\circ} \mathrm{C}$ for $15-60$ minutes, rinsed briefly in physiological saline, fixed in $10 \%$ formalin for 24 hours, passed through graded alcoholic solution and xylol and mounted in Canada balsam.

The final concentration of the components of medium was according to Nachlas et $\mathrm{al}^{12}$, 2), except for sodium glyceraldehyde-3-phosphate, sodium L-gulonate, DPNH and TPNH. The optimal concentration of these four reagents was $0.25 \mathrm{M}, 0.5 \mathrm{M}, 4.5 \mathrm{mM}$ and $4.5 \mathrm{mM}$, respectively.

\section{Results and discussion}

(1) On the basis of the finding that DPNH- or TPNH-TR activity is much more intense than DPN- or TPN-linked dehydrogenase activity tested above in the cytoplasm of all of the structural components of the human skin, it can be said that the former is not a rate-limiting factor in the hydrogen transfer from substrate to Nitro-blue tetrazolium, that is, the latter is responsible for the intensity of reduction of Nitro-blue tetrazolium.

(2) Distribution of the various dehydrogenases related to glucose metabolism in the human skin can be divided into the following three patterns. 
A) Form I; SDH and DPN-ICDH take this form. It is characterized by the following. In the epidermis (Fig. 1), there is a gradual reduction of activity from the str. basale towards the str. granulosum. Activity is highest in the eccrine sweat duct (Fig. 1-ED, Fig. 12-D), especially in the basal cell, but almost absent in the cuticular border (Fig. 8). The transitional zone (Fig. 12-T) between the duct and secretory segment (Fig. 12-S) is clearly defined. In the sebaceous gland (Fig. 1), activity decreases as the cells mature. Distribution of enzyme activity corresponds quite colsely to the density of the mitochondira ${ }^{3)}$. The weak activity in the matrix and bulb (Fig. 6) is in proportion to the activity of mitochondria in these areas. It can be said without doubt that SDH is a mitochondrial enzyme, and DPN-ICDH is believed to be exclusively localized intramitochondrially. Both are members of the TCA cycle. It is therefore suggested that this form shows a distribution of mitochondrial portion of TCA cycle. It corresponds to the site of oxidative phosphorylation under physiological conditions. It is clear from the histochemical pattern that mitochondrial enzymes are closely related to cellular proliferation, but the enzyme activity does not always parallel cell division. For example, the hair matrix is mitotically active, but not active enzymatically. The eccrine sweat duct, on the other hand, is not active mitotically, but extremely active enzymatically. Such a feature can be seen between the methylcholanthrene-induced skin cancer and the epidermal hyperplasia surrounding the cancer. SDH activity is rather reduced in the former and highly increased in the latter ${ }^{4}$.

B) Form II ; G6PDH, 6PGDH, TPN-ICDH and ME belong to this category. It is characterized by the following. There is an increase in enzymatic reaction in the epidermis (Fig. 3,4) upward and it is maximal in the str. granulosum (Fig. 4). Of the tissues, reaction is highest in the sebaceous gland (Fig. 3-S) and it increased toward the lumen as the cells mature. Reaction is rather low in the excretory duct of the sweat gland but outstandingly increased in the cuticular border (Fig. 7, 4). A marked activity is present in the keratogenous zone (Fig. $5-\mathrm{CH}$ ) of the cuticle of the hair. The distribution appears opposite that of Form I. A similar form is seen in the case of $\beta$ $\mathrm{HBDH}$. From the distribution pattern, it is believed that TPN-ICDH, as well as G6PDH and 6PGDH, play an important role in the production of TPNH in the extramitochondrial compartment. As to the human skin, DPN-ICDH is probably more active than TPN-ICDH as a member of TCA cycle, and TPNICDH intensely active as a member of TPNH production system. Activity of the TPNH production system increases outerward from the str. basale in the epidermis, centrally in the sebaceous gland, axially in the excretory duct of sweat gland and outer root sheath and in the keratogenous zone of hair cuticle, that is, activity increases with maturation of the epithelial cells of the skin. It has been observed previously that with maturation, lipogenesis proceeds in the sebaceous gland, keratogenesis probably together with lipogenesis in the epidermis and keratogenesis in the outer root sheath, cuticle of hair and eccrine sweat duct $^{(3), 5), 6)}$. It can, therefore, be assumed that there is a 


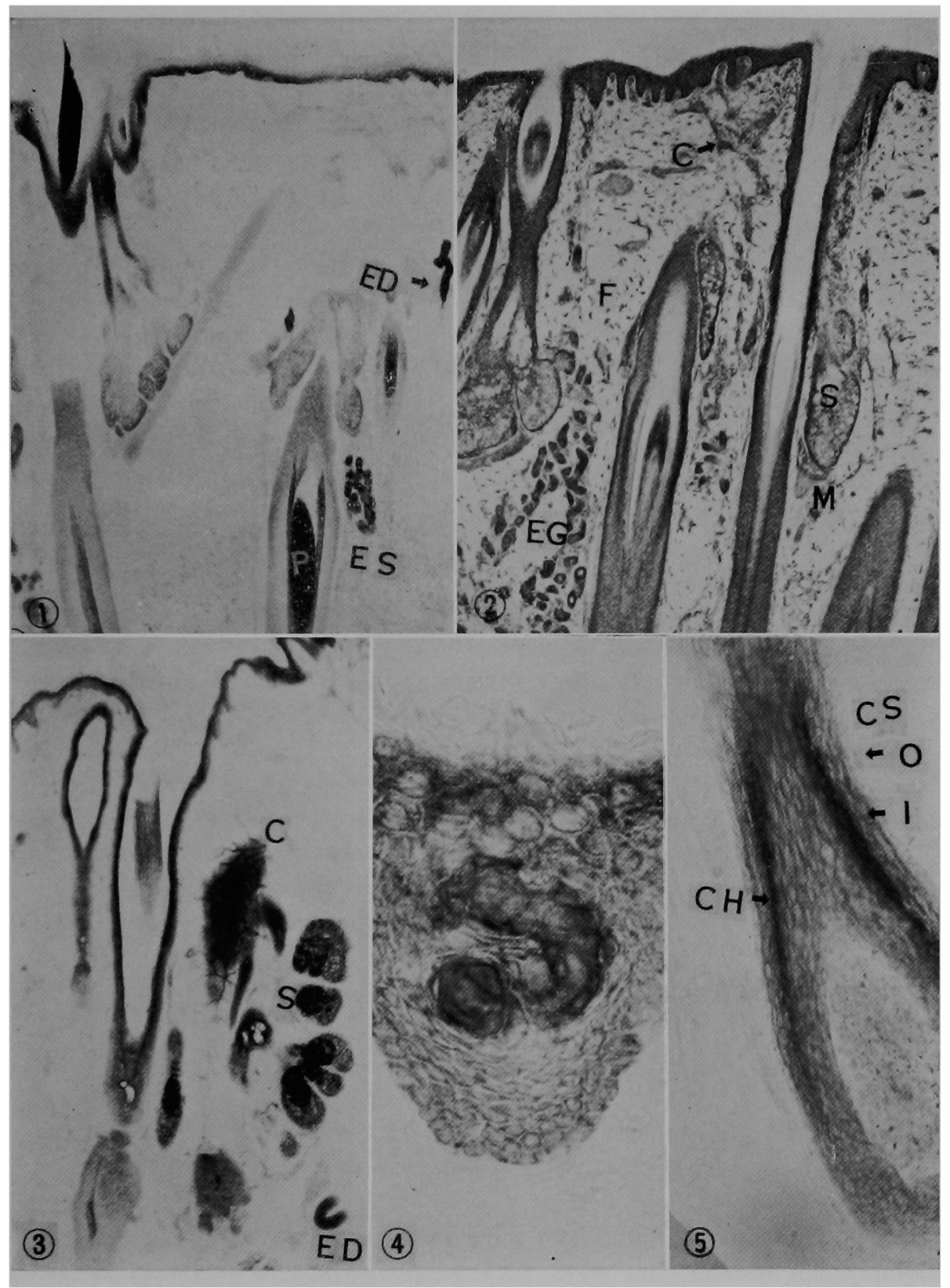

great possibility that lipogenesis and keratogenesis of the skin are related to TPNH production. In fact, it has been shown biochemically that lipogenesis is TPNH-dependent in the extramitochondrial compartment ${ }^{7)}$. It is interesting that, of the TPN-liked dehydrogenases of skin which were tested, only TPN$\mathrm{ICDH}$ is markedly active in the melanocytes (Fig. 10-M). Experiments in the mouse have, moreover, shown that activation of melanocytes by painting the skin with 20 -methylcholanthrene results in a pronounced increase in enzyme activity (Fig. 11-M). These suggest that TPN-ICDH is involved in melano- 


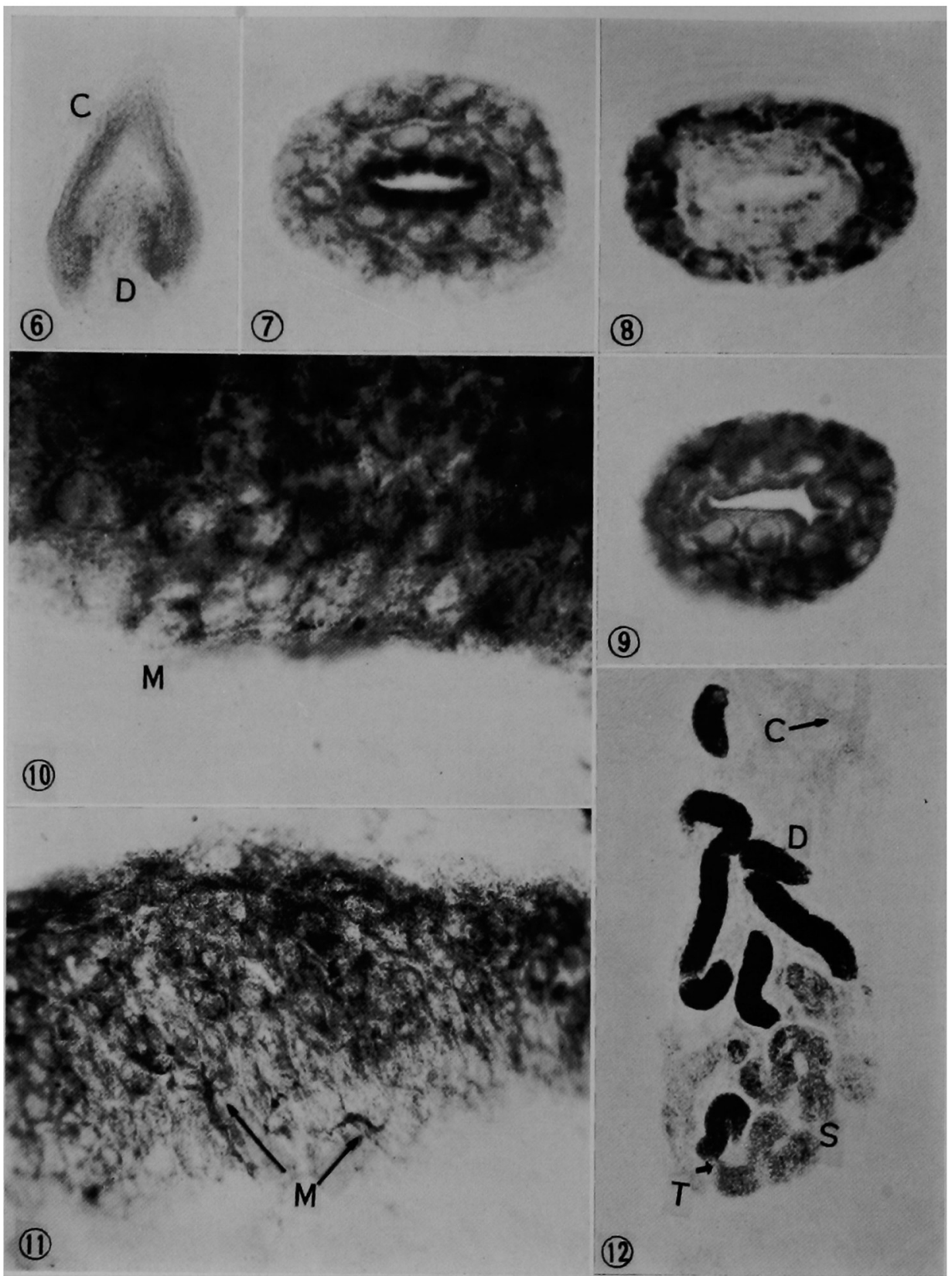

genesis. From the finding in vitro experiments that the process, phenylalanine $\rightarrow$ tyrosine is TPNH-dependent ${ }^{\text {H) }}$, it may be assumed that TPN-ICDH is coupled with this process.

C) Form III; This is the form demonstrated with GA3PDH and LDH. The cell elements in the skin show a strong reaction in general and the difference in the reaction between the structural components is less than in Form I and II (Fig. 2, 9). As this form is demonstrated with GA3PDH and 
$\mathrm{LDH}$, it is believed to show a distribution pattern of EM glycolysis. A similar form is obseved, however, in the case of $\mathrm{MDH}$. It has been recently shown that there are two types of this enzyme; a $\mathrm{M}-\mathrm{MDH}$ in mitochondrion and a

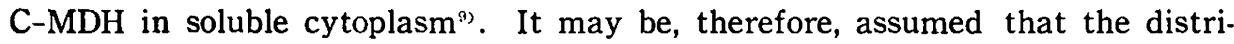
bution pattern of malic dehydrogenase appears similar to Form III by a combination of the Form I due to the M-MDH probably related to the other mitochondrial enzymes of TCA cycle and Form II induced by the C-MDH probably related to Ochoa's malic enzyme and other soluble enzymes.

In the eccrine sweat duct, the mitochondrial enzymes of TCA cycle and the two glycolysis enzymes show a high activity. From the viewpoint of ATP production, these facts suggest that active transport for reabsorption may take place in this tissue.

D) Although the epithelial tissue of the skin takes a highly differentiated complex morphology, it may be considered as a stratified structure which matures from the deep layer next to the corium, outward toward the superficial layer. When the structure of the skin is viewed in this way, the distribution of dehydrogenases related to glucose metabolism of the skin may be divided into the following 3 patterns, they are, the "deep layer pattern" of oxidative phosphorylation (Form I), the "superficial layer pattern" of TPNH production and reductive biosynthesis (Form II), and the "total layer pattern" of EM glycolysis (Form III). From this, it may be said that from the deep layer toward the superficial layer, aerobic energy production decreases with relative increase in glycolysis, and that increased utilization of energy involving TPNH induces progressive maturization, e.g. keratogenesis, lipogenesis and other biological synthesis, of the epithelial tissue of the skin.

E) The finding that $\beta-\mathrm{HBCH}$ shows a distribution pattern of activity similar to TPN-linked dehydrogenases may be explained by the relationship between ketone body and lipid in intermediary metabolism, but this distribution pattern appears contrary to the results obtained previously at the subcellular level, that this is a mitochondial enzyme. It is believed in general, that GDH is a mitochondrial enzyme, but the distribution pattern of activity at the cellular level differs in part from that of TCA cycle members as SDH and DPN-ICDH. If the previous concept that these two enzymes are localized solely in the mitochondria is accepted, it must be said that the specific distribution patterns of these enzymes show a heterogeneity of mitochondria.

DPN-GuDH and TPN-GuDH are more weakly active compared to dehydrogeanases belonging to EM glycolysis, TCA cycle and Hexose monophosphate shunt.

\section{Literature}

1) Nachlas, M. M., et al. : A histochemical method for the demonstration of diphosphopyridine nucletide diaphorase. J. Histochem. Cytochem., 4:29, $1958 \quad$ 2) Nachlas, M.M., et al.: The histochemical localization of triphosphopyridine nucleotide diaphorase. J. Bi. ophy. Biochem. Cytol., 4:467, 1958. 3 3) Montagna, W.: The structure and fuction of skin. Academic Press Inc. Publishers, New York, 1956. 4) Hashimoto, T. : unpublished. 5) Ellis, R. A., et al. : Electron microscopy of the duct and especially the cuticular border 
of the eccrine sweat glands in Macaca HuLaTTA. J. Biophys. Biochem. Cytol., 9: 238, 1961. 6) Montagna, W., et al.: Dedifferentiation of sebaceous glands in the mouse after total extirpation with methylcholanthrene. Anat. Rec., $107: 83,1.7$ ) Kingenberg, M., et al. : Biological oxdations. Ann. Rev. Biochem., $29: 669,1960 . \quad$ 8) Kaufman, S. : The enzymatic conversion of phenylalanine to tyrosine. J. Biol. Chem., $226: 511,1957$. 9) Grimm, F.C., et al.: Properties of the two forms of malic dehydrogenase from beef heart. J. Biol. Chem., 236: 1980, 1961.

\title{
Discussion
}

Dr. Yasuda :

1) The keratogenous zone often shows non-specific staining through the tetrazolium reduction. How was it in your present studies?

2) The crust of epidermal sweat duct which contain much sticky substance easily results in nonspecific staining by various histochemical reactions. How was your present observation?

3) How were the activities in myoepithelium of the sweat glands ?

Dr. Hashimoto :

1) Of hair cortex, the keratogenous zone may causes the nonspecific formazan deposition, but not of hair cuticle.

2) The epidermal sweat ducts show only specific reactions to some TPN-linked dehydrogenases.

\section{Histochemical Studies on the Damaged Liver}

\author{
Shigetoshi Ohkawa, Yoshiaki Okuda, Akima Mryoshi \\ and Gyoichi Wakisaka. \\ The First Division, Department of Internal Medicine, \\ Faculty of Medicine, Kyoto University.
}

Histochemical studies of the enzymes in the damaged liver have been successively performed by Wachstein et al. (1958) ${ }^{12}$. On the other hand, heterogeneity of enzymic activity within the liver lobule was pointed out histochemically by Novikoff $(1959)^{2)}$ and microchemically by Shank et al. (1957) ${ }^{3)}$.

In the present study liver injury was induced in animals and the histochemical findings of the liver at the various stages of the injury were successively investigated.

\section{Materials and Methods}

Acute intoxication of the liver was induced with $\mathrm{CCl}_{4}$ in rabbits and $\mathrm{DL}-$ ethionine in rats. In addition, partial hepatectomy was made in rats, according to the method of Higgins \& Anderson $(1931)^{4)}$. The animals were sacrificed by exsanguination and small blocks of the liver were promptly freezed by dry-ice. These blocks were cut in 15 microns in thickness by cryostat at $-18^{\circ} \sim$ $-15^{\circ} \mathrm{C}$ and the following enzymes were stained. 